\title{
Auswirkungen von Budgets auf die ambulante Versorgung
}

\author{
Nora Willea, Jürg Schlup ${ }^{b}$ \\ ${ }^{a}$ Dr. phil., persönliche wissenschaftliche Mitarbeiterin des Präsidenten; ${ }^{b}$ Dr. med., Präsident der FMH
}

\begin{abstract}
Aktuell werden in der Schweiz vielfach «Kostenbremsen» oder explizit "Globalbudgets» für die ambulante Versorgung gefordert. Wie sich solche Budgets im Praxisalltag in Deutschland niederschlagen, war im letzten Jahr in einigen deutschen Medien nachlesbar.
\end{abstract}

Im Herbst 2017 machte in Deutschland der Allgemeinmediziner und Landarzt Dr. med. Günter Krause Schlagzeilen, als er ankündigte, seine Zulassung als Kassenarzt - also seine Berechtigung zur Abrechnung mit der gesetzlichen Krankenversicherung - öffentlich zu verbrennen. Der 38-Jährige, der als «gemütlicher Typ mit robustem Humor» [1] beschrieben wird, war nach Medizinstudium und Facharztausbildung in seinen 1500 Einwohner zählenden Heimatort Hainewalde zurückgekehrt und hatte nach einigen Jahren der $\mathrm{Zu}$ sammenarbeit 2014 die Landarztpraxis seiner Mutter übernommen. Im Oktober 2017 schien ihm seine berufliche Existenz angesichts der miserablen Arbeitsbedingungen jedoch nichts mehr zu sein, das sich zu erhalten lohnt. Er schrieb einen Brandbrief und wollte seine Praxis schliessen. Sein Protest traf einen Nerv: Kollegen und Kolleginnen bekundeten ihre Solidarität, und auch die Medien berichteten, zunächst lokal in der Sächsischen Zeitung [2], später auch überregional in der Zeit [1].

\section{Die Wurzel des Problems: Behandlungen nach planwirtschaftlichen Vorgaben}

Die Probleme der Gesundheitsversorgung, die in diesen Berichten beschrieben werden, entstehen durch die in Deutschland praktizierte Budgetierung der ambulanten Versorgung. Denn Dr. Krause darf seinen rund 1100 Patienten, darunter die Bewohner von rund einem Dutzend Altersheimen, nur so viele Versorgungsleistungen zukommen lassen, wie das ihm vorgegebene Budget erlaubt. Diese «planwirtschaftlichen Vorgaben" [2] basieren auf Durchschnittswerten, sind aber häufig vor Ablauf des Zeitraums bereits aufgebraucht, wenn die Erkrankungen der Patienten eben nicht verlaufen, wie es sich die Kostenträger wün- schen. Überschreitungen des Budgets muss Dr. Krause aus eigener Tasche finanzieren.

Wie sich das im Alltag von Dr. Krause niederschlägt, beschreibt er so: «Wir verschwenden unsere Zeit für die Bürokratie und streiten uns ständig mit der Kassenärztlichen Vereinigung herum» [2]. Denn die unter ministerialer Aufsicht stehenden Kassenärztlichen Vereinigungen (KVen), denen alle Vertragsärzte angehören müssen, sind für die Verteilung der von den Krankenkassen geleisteten Gesamtvergütung zuständig. Die KV muss also das Gesamtbudget, das im Wesentlichen durch den Beitragssatz aller Pflichtversicherten von $14,6 \%$ des Bruttoeinkommens vorgegeben ist, auf die einzelnen Ärzte verteilen und ihre Ansprüche prüfen.

\section{Administrativer Overkill zur Recht- fertigung ärztlicher Entscheide}

Für Dr. Krause hatte dies zur Folge, dass seine etwa zehnstündigen Arbeitstage im Jahr 2017 um drei Kassenprüfungen ergänzt wurden, bei denen er z.B. schriftlich darlegen muss, "warum die Stützstrümpfe, die Physiotherapie oder die verschriebenen Tabletten für Patientin X nötig waren» [2]. Kann er die Notwendigkeit geleisteter Behandlungen oder verschriebener Medikamente nicht hinreichend belegen, muss er Rückzahlungen leisten: «Ich bezahle Behandlungen, die ich vor dreieinhalb Jahren durchgeführt habe, jetzt aus eigener Tasche» [2], berichtete Dr. Krause der Sächsischen Zeitung. Ob er als behandelnder Arzt Medikamente und Therapien als sinnvoll beurteilt, ist zweitrangig - entscheidend sind die Auffassungen von Krankenkassen und KV. Selbst seine Mutter, von der er die Praxis 2014 übernommen hat, sei als Rentnerin noch mit fünfstelligen Regressforderungen der Kassen konfrontiert. Der Motivationsverlust von Ärzten und Ärztinnen ist leicht 
nachvollziehbar, wenn ihre Behandlungsentscheide fortwährend im Lichte planwirtschaftlicher Vorgaben in Frage gestellt werden. Dr. Krause betrachtet darum auch die Therapiefreiheit als nicht existent: "Ich verschreibe Medikamente doch nicht zum Spass» [2]. Er befürchtet, "dass die medizinische Versorgung durch Landärzte bald zusammenbricht» [2] - was plausibel erscheint, wenn selbst ein in seinem Heimatdorf verwurzelter Landarzt wie Dr. Krause Lust hat, alles hinzuschmeissen.

\section{Begrenztes Budget $=$ Regressforderungen und unbezahlte Arbeit}

Die durch die Budgetierung verursachten Probleme betreffen aber nicht nur Landärzte und Grundversorger: Die Krankenkassen «würden für einen Grossteil der ärztlichen Leistungen nur ein bestimmtes Budget zur Verfügung stellen. Das aber würde nicht ausreichen, alle Leistungen der niedergelassenen Ärzte in vollem Umfang zu vergüten" [1], liess sich der Sprecher der KV Sachsen in der Sächsischen Zeitung zitieren und bezog sich dabei auf den Fall "eines Orthopäden, der wegen Budgetüberschreitung zeitweise unentgeltlich arbeitete» [1].

In dieser Weise äusserte sich auch Dr. med. Andreas Gassen, Vorstandsvorsitzender der Kassenärztlichen Bundesvereinigung und Orthopäde. In einem Interview mit dem Spiegel berichtete er, dass «im Paxisalltag [...] die starren Budgets tatsächlich dazu [führen], dass ein Arzt genau rechnen muss, was er verordnen und wie viele Patienten er sich leisten kann" [3]. Seine Forderungen zeigen, was unter dem Globalbudget nicht mehr als selbstverständlich gilt: «Wir wollen einfach nur, dass jeder Besuch eines Patienten honoriert wird, dass vor allem bei jeder Erstkonsultation die Grundpauschale für jeden Patienten gezahlt wird" [3]. Da auch in seiner eigenen Praxis jeder achte Patient "quasi unentgeltlich» [3] behandelt werden muss, äussert er Verständnis dafür, "wenn Kollegen in einer ähnlichen Situation dreimal überlegen, ob sie noch die letzten Patienten annehmen, die um fünf vor fünf vor der Praxistür warten, wenn das Budget schon ausgeschöpft ist» [3]. Die Budgetierung betrifft aber nicht nur Akutpatienten vor der Praxistür, sondern spiegelt sich auch in den Wartefristen für Termine, die z.B. für einen Kardiologentermin in Hamburg schon 71 Tage betrugen - für Privatpatienten 19 [4]

\section{Budgetierung als Basis der Zwei-Klassen- Medizin}

Die Budgetierung in der ambulanten Versorgung betrifft ausschliesslich die Versicherten gesetzlicher Krankenversicherungen - also etwa 90\% der deutschen Bevölkerung. Wer privat bezahlt oder privat versichert ist, kann ohne Budgetrestriktionen behandelt werden. An der resultierenden Zwei-Klassen-Medizin lassen sich somit indirekt die Auswirkungen der Budgetierung ablesen. So berichtet z.B. Orthopäde Gassen, dass aus medizinischer Sicht sinnvolle Massagen, z.B. bei Spastiken oder schmerzhaften Muskelerkrankungen, bei Privatversicherten problemlos verordnet werden können. "Bei einem gesetzlich Versicherten muss ein Arzt zwangsläufig darauf achten, ob er mit einer Verordnung sein Budget überschreitet - und damit auch für die Behandlung aller anderen Patienten keine Leistungen mehr verordnen kann, ohne in einen Regress zu laufen" [3].

Als Antwort auf die Frage des Spiegels, wie man das Gesundheitssystem aus seiner Sicht gerechter machen könnte, stellt Dr. Gassen entsprechend fest: «Ganz einfach: Man sollte die Budgets in der gesetzlichen Krankenversicherung aufheben" [3]. Als Fazit aus den Erfahrungen des Landarztes Dr. Krause und des KV-Vorstands und Orthopäden Dr. Gassen lässt sich also festhalten: Für eine bessere und gerechte Versorgung in Deutschland müsste das Prinzip aufgehoben werden, dessen Einführung uns in der Schweiz aktuell als Kostenbremse ohne schädliche Nebenwirkungen angepriesen wird.

\section{Literatur}

1 Reinhard D. Ihm reicht es. Die Zeit. 17.11.2017.

2 Heinke M. Landarzt will seine Zulassung verbrennen. Sächsische Zeitung. 28.10.2017.

3 Interview mit Andreas Gassen im Spiegel 7/2018, S. 65-6; Titel: "Das ist eine Tortur».

4 Spiegel online. 17.7.2011. Ärzte diskriminieren Kassenpatienten systematisch. 